\title{
Preparation and evaluation of microemulsion-based transdermal delivery of Cistanche tubulosa phenylethanoid glycosides
}

\author{
JIANHUA YANG ${ }^{1 *}$, HUANHUAN XU ${ }^{1-3^{*}}$, SHANSHAN WU ${ }^{2}$, BOWEI JU ${ }^{2}$, \\ DANDAN ZHU ${ }^{2}$, YAO YAN ${ }^{1}$, MEI WANG $^{2}$ and JUNPING HU ${ }^{2}$ \\ ${ }^{1}$ Department of Pharmacy, The First Affiliated Hospital; ${ }^{2}$ Department of Natural Medicines, College of Pharmacy, \\ Xinjiang Medical University, Urumqi, Xinjiang 830011; ${ }^{3}$ Department of Pharmacy, \\ Kanghua Hospital, Dongguan, Guangdong 523003, P.R. China
}

Received December 2, 2015; Accepted November 29, 2016

DOI: $10.3892 / \mathrm{mmr} .2017 .6147$

\begin{abstract}
The primary aim of the present study was to develop a novel microemulsion (ME) formulation to deliver phenylethanoid glycoside (PG) for use in skin lighteners and sunscreens. The oil phase was selected on the basis of drug solubility, while the surfactant and cosurfactant were screened and selected on the basis of their solubilizing capacity and the efficiency with which they formed MEs. Pseudoternary phase diagrams were constructed to evaluate ME regions and five formulations of oil-in-water MEs were selected as vehicles. In vitro skin permeation experiments were performed to optimize the ME formulation and to evaluate its permeability in comparison to that of saline solution. The physicochemical properties of the optimized ME and the permeating ability of PG delivered by this ME were also investigated. The optimized $\mathrm{ME}$ formulation was composed of isopropyl myristate (7\%,w/w), Cremorphor EL (21\%,w/w), propylene glycol $(7 \%$, $\mathrm{w} / \mathrm{w})$ and water $(65 \%, \mathrm{w} / \mathrm{w})$. The cumulative amount of PG that permeated through excised mouse skin when carried by ME was $\sim 1.68$ times that when PG was carried by saline solution only. The cumulative amount of PG in the microemulsion $\left(4149.650 \pm 37.3 \mu \mathrm{g} \cdot \mathrm{cm}^{-2}\right)$ was significantly greater than that of PG in the saline solution $\left(2288.63 \pm 20.9 \mu \mathrm{g} \cdot \mathrm{cm}^{-2}\right)$. Furthermore, the permeability coefficient indicated that optimized microemulsion was a more efficient carrier for transdermal delivery of PG than the control solution $\left(8.87 \pm 0.49 \mathrm{~cm} / \mathrm{h} \times 10^{-3}\right.$ vs. $\left.5.41 \pm 0.12 \mathrm{~cm} / \mathrm{hx} 10^{-3}\right)$. Taken together, the permeating ability of ME-carried PG was significantly increased compared with saline solution.
\end{abstract}

Correspondence to: Dr Junping $\mathrm{Hu}$, Department of Natural Medicines, College of Pharmacy, Xinjiang Medical University, 393 Xinyi Road, Urumqi, Xinjiang 830011, P.R. China

E-mail: hujp0551@yeah.net

*Contributed equally

Key words: phenylethanoid glycoside, microemulsion, transdermal delivery, skin lightener, sunscreen

\section{Introduction}

Herba cistanche, a tonic herb commonly distributed in desert regions, has been frequently used in traditional Chinese medicine. To date, multiple studies have been conducted to investigate its biopharmaceutical properties, including improvement of memory, sexual potency, free radical scavenging, and anti-aging activity (1). The major component of $H$. cistanche is phenylethanoid glycoside (PG). Previous studies have revealed that PG inhibits the activity of tyrosinase, the rate-limiting enzyme for the synthesis of melanin in the human body (2). In addition, PG has been reported to be responsible for the scavenging of 2,2-diphenyl-1-picrylhydrazyl radicals (3), and protecting keratinocytes from ultraviolet (UV) radiation. Therefore, this component may have potential in skin depigmentation.

Topical medication has been commonly used in cosmetic fields, with the drug delivered directly onto the body surface (4). Microemulsions (ME) are a novel drug delivery system conventionally composed of oil, surfactant, cosurfactant and aqueous phases (5) and have been reported to improve the rate and absorption of lipophilic drugs (6). In addition, due to its satisfactory solubility, low skin irritation and high permeability, it is expected to be used in topical drug delivery $(7,8)$.

To the best our knowledge, few studies have been conducted where PG was delivered via topical medication using microemulsion. In the present study, an oil-in-water $(\mathrm{O} / \mathrm{W})$ nanoemulsion delivery system for transdermal administration was established, and PG was incorporated into the nanoemulsion $(9,10)$. Meanwhile, preparation and characterization of PG-based nanoemulsion was performed by investigating the transdermal release in vitro.

\section{Materials and methods}

Reagents. Cremorphor EL and isopropyl myristate (IPM) were purchased from Sigma Aldrich; Merck Millipore (Darmstadt, Germany). Ethanol, oleic acid (OA) and Tween-80 were purchased from Guangcheng Chemical Reagent Co., Ltd. (Tianjin, China). Ethyl oleate was purchased from Shanghai Chemical Reagent Co., Ltd. (Shanghai, China). All chemicals and solvents used were of analytical reagent grade. 
Preparation of PhGs. PhG was prepared as routinely described (11). The air-dried C. tubulosa was powered and extracted by percolation with $80 \% \mathrm{EtOH}$. The percolate was evaporated under pressure, followed by filtration of the residual fluid. The filtrate was concentrated and chromatographed on a SP-825 macroporous resin column using 0, 30, 50, 70 and 90\% EtOH in water as eluants. The $30-50 \%$ EtOH eluants were concentrated and dried to obtain $\mathrm{PhG}$ rich fraction. UV spectrophotometry indicated the content of the $\mathrm{PhG}$ was $87.6 \%$.

Screening of oils, surfactants and cosurfactants for ME. Solubility tests were conducted to select appropriate components for the preparation of an ME formulation with a high drug-loading capacity. In this process, an excess amount of PG was added into $4 \mathrm{ml}$ of each of the following reagents: IPM, liquid paraffin, OA, Cremophor EL, Tween-80, absolute ethanol, propylene glycol, glycerin and PEG400, respectively. Following this, the resulting mixture was shaken reciprocally at $37^{\circ} \mathrm{C}$ for $72 \mathrm{~h}$, followed by centrifugation at $12,830 \times \mathrm{g}$ for $10 \mathrm{~min}$. Subsequently, the pellet was resuspended in methanol, and the PG was determined using UV spectrophotometry at $333 \mathrm{~nm}$. Appropriate diluted solutions of oils, surfactants and cosolvents in methanol served as a blank control.

Construction of phase diagram. To obtain a concentration range of components for the existing microemulsion boundary, pseudoternary phase diagrams were constructed using a water titration method as previously described (12). Three phase diagrams were prepared with 1:3, 1:1, and 3:1 weight ratios of surfactant to cosurfactant. The oil phase and the surfactant mixture were then mixed at the weight ratios of 1:9, 2:8, 3:7, 4:6, 5:5, 6:4, 7:3, 8:2 and 9:1. The oil and surfactant mixture were mixed homogeneously, and were diluted dropwise with purified water under magnetic stirring (300 rpm) at room temperature until transparent ME was obtained. The concentration of components was recorded in order to complete the pseudoternary phase diagram. Based on these diagrams, the appropriate concentrations of materials were selected for the preparation of PG-based ME.

Preparation of ME and the aqueous solution of $P G$. A total of 5 MEs (ME1-5) were selected according to the phase diagrams with a surfactant: Cosurfactant weight ratio of 3:1 (Table I). Microemulsion systems were prepared by mixing oil with the mixture of surfactant and cosurfactant, and water was added precisely into oily phases with magnetic stirring (300 r/min) at $37^{\circ} \mathrm{C}$. The systems were equilibrated using gentle magnetic stirring for $30 \mathrm{~min}$ followed by dissolving the appropriate amount of PG under ultrasonication. The aqueous solution was prepared with the same PG content dissolved in purified water.

Characterization of microemulsion. The average droplet size and polydisparity index of MEs were evaluated by photon correlation spectroscopy (Malvern Instruments, Ltd., Malvern, UK). All samples were diluted with water at a 1:5 ratio and were filtered through a $0.22 \mu \mathrm{m}$ filter. The test was performed in a thermostatic chamber at $25^{\circ} \mathrm{C}$ in triplicate.

The morphology of PG microemulsions was observed using transmission electron microscopy (JEM-100CXII; JEOL Ltd., Tokyo, Japan). Briefly, one drop of diluted sample was deposited on a film-coated coppergrid, followed by staining with one drop of $2 \%$ phosphotungstic acid aqueous solution. The average droplet diameters of the microemulsions were determined by photon correlation spectroscopy instrument (BI-200SM; Brookhaven Instruments Corporation, Holtsville, NY, USA) at room temperature.

The viscosity of the ME vehicles was measured at $25^{\circ} \mathrm{C}$, using the NDJ-8S digital viscometer (Shanghai Precision and Scientific Instrument Co., Ltd., Shanghai, China) with a no. 1 rotor set at $60 \mathrm{rpm}$.

$p H$ determination. The $\mathrm{pH}$ values of the MEs were determined at $25^{\circ} \mathrm{C}$ using the Phs-3C digital acidimeter (Shanghai REX Instruments Factory, Shanghai, China). The refractive indices were measured with a thermostatic Abbe refractometer (Optical Instrument Factory, Shanghai, China).

Preparation of skin tissues. Male Kunming mice weighing $20 \mathrm{~g}$ were purchased from Experimental Animal Center of Xinjiang Medical University (Xinjiang, China). 10 mice (6 weeks old) were subjected to adaptive feeding for 1 week on $12 \mathrm{~h}$ light $/ 12 \mathrm{~h}$ dark cycle, at room temperature $\left(24 \pm 2^{\circ} \mathrm{C}\right)$, with a humidity of $50 \pm 15 \%$. All animals had access to a standard diet and water. The hair was removed with an electrical clipper. Following terminal anesthesia with chloral hydrate (10\%) via subcutaneous injection, skin tissues were excised from the abdominal region and the subcutaneous fat and connective tissue were removed. The tissues were placed in a refrigerator at $4^{\circ} \mathrm{C}$ overnight. The study protocols were approved by the Ethical Committee of Xinjiang Medical University (Xianjiang, China).

In vitro permeation study. Permeation experiments were conducted as previously described (12). The excised skin was clamped between the donor and the receptor chamber of a vertical diffusion cell with an effective diffusion area of $2.8 \mathrm{~cm}^{2}$. The receptor chamber was filled with fresh, normal saline. The diffusion cell was maintained at $37^{\circ} \mathrm{C}$ using a recirculating water bath and the solution in the receptor chambers was stirred continuously at $200 \mathrm{rpm}$. Following this, the test formulation $(1.0 \mathrm{ml})$ was gently placed in the donor chamber. Subsequently, $3 \mathrm{ml}$ of the solution in the receptor chamber was removed and replaced with an equal volume of fresh normal saline at predetermined intervals $(0,2,4,6,8,10$ and $24 \mathrm{~h})$. The sample obtained from the receptor chamber was used to determine PG levels using UV spectroscopy at a wavelength of $333 \mathrm{~nm}$.

The cumulative amount of PG that permeated through the excised mouseskins was calculated according to the following formula:

$$
Q n=\frac{\left(C_{n}+\frac{V}{V_{0}} \sum_{i=1}^{n-1} C i\right) V_{0}}{S}
$$

Where $Q_{n}$ was the accumulated amount at a given time $t$, $\mathrm{Cn}$ is the drug concentration of the receiver solution at each sampling time, $\mathrm{C}_{\mathrm{i}}$ is the drug concentration of the sample, $\mathrm{S}$ is the effective diffusion area $\left(S=2.8 \mathrm{~cm}^{2}\right)$, and $V_{0}$ and $V_{i}$ are the volumes of the receiver solution and the sample, respectively. 
The cumulative amount $\left(Q_{n}\right)$ of PG permeated was plotted as a function of time $(t)$ for each formulation. The permeation rate of PG at a steady-state $\left(\mathrm{J}_{\mathrm{ss}}, \mu \mathrm{g} / \mathrm{cm}^{-2} / \mathrm{h}^{-1}\right)$ through the mouse skin was calculated from the slope of linear portion of the plots of Qnversus time. The permeability coefficient $\left(\mathrm{K}_{\mathrm{p}}, \mathrm{cm} / \mathrm{h}\right)$ was calculated according to the following formula:

$$
K_{p}=\frac{J_{S S}}{C_{0}}
$$

Where $\mathrm{K}_{\mathrm{p}}$ is the permeability coefficient, $\mathrm{J}_{\mathrm{ss}}$ is the flux calculated at steady-state, and $\mathrm{C}_{0}$ represents the drug concentration which remained constant in the vehicle.

Skin retention study. The following sections detail the comparison of the ex vivo permeation behavior, cumulative amount, permeation rate, permeability coefficient and skin retention characteristics between optimized ME formulations (PG content, 2\%, w/w) and the aqueous solution.

At the end of the permeation experiment, the residual drug level in the skin was determined using a homogenization method. Following washing with normal saline, the skin was cut into small sections and placed in a glass tube containing $3 \mathrm{ml}$ methanol in an ice bath. The sample was homogenized with a speed of $12000 \mathrm{r} / \mathrm{min}$. rpm for $2 \mathrm{~min}$, and then centrifuged at $12,830 \times \mathrm{g}$ horizontally for $30 \mathrm{~min}$ at $25^{\circ} \mathrm{C}$. The supernatant was passed through a microporous filter $(0.45 \mu \mathrm{m})$, and the PG levels in the supernatant were determined by UV spectroscopy at a wavelength of $333 \mathrm{~nm}$.

Stability study. The physical stability of PG-loaded ME was investigated via clarity, particle size analysis and phase separation, which was observed at $2-8^{\circ} \mathrm{C}$ and at room temperature for 3 months. Centrifugation was performed at $12,830 \times \mathrm{g}$ for $30 \mathrm{~min}$ at $25^{\circ} \mathrm{C}$ to assess the physical stability of ME. Phase separation and concentration of PG were investigated monthly to judge the optimal storage temperature. Chemical stability was evaluated on drug loaded formulations, stored at $2-8^{\circ} \mathrm{C}$ and at room temperature, with the PG content determined by UV spectrophotometry at $333 \mathrm{~nm}$.

Skin sensitivity test. Skin sensitivity tests were performed on rabbits (weighing $2.02 .5 \mathrm{~kg}$ ) to evaluate the irritant potential of topical application of the developed formulation as previously described (13). Nine Holland rabbits (16 weeks old; weighing 2.0-2.5 kg; 5 male and 4 female) were obtained from the Experimental Animal Center of Xinjiang Medical University. The animals were subjected to adaptive feeding for 1 week on $12 \mathrm{~h}$ light $/ 12 \mathrm{~h}$ dark cycle, at room temperature $\left(24 \pm 2^{\circ} \mathrm{C}\right)$, with a humidity of $50 \pm 15 \%$. All animals had access to a standard diet and water. Air embolism was used for the sacrifice. The protocols used in the present study were approved by the Ethic Committee of Xinjiang Medical University (Xinjiang, China). The hairs on the back were removed $\sim 24 \mathrm{~h}$ prior to administration of the ME formulations. Animals were divided into the following groups: An optimized ME group ( $n=3)$, with topical administration of optimized ME containing 2\% (w/w) PG; a PG saline group $(n=3)$, with topical administration of saline solution containing $2 \%(\mathrm{w} / \mathrm{w}) \mathrm{PG}$; and control saline group $(n=3)$, subject to topical administration of normal saline. The administration was performed twice a day for 5 days. The animals were observed for any signs of itching or changes in the skin, including erythema, papule, flakiness and dryness (14). Following drug withdrawal, observation for single or multiple administrations was continued for 3 days. The irritation scores of the test area were evaluated by judging the extent of erythema and edema according to the following criteria: 1 , no visible reaction; 2 , a slight reaction; 3 , a moderate reaction and 4 , a severe reaction $(15,16)$. Finally, the total irritation scores for each condition were calculated using the following equation:

Averaged irritation scores $=($ erythema reaction + edema reaction) / number of animals

Statistical analysis. Data are presented as the mean \pm standard deviation. Data analysis was performed using one-way analysis of variance followed by the least significant difference test. Data were analyzed using SPSS software version 19.0 (IBM SPSS, Armonk, NY, USA). P $<0.05$ was considered to indicate a statistically significant difference.

\section{Results and Discussion}

Screening of oils, surfactants and co-surfactants for ME.PG was almost insoluble in liquid paraffin and oleic acid, but moderate solubility was observed in IPM (Table II). IPM increases the diffusion coefficient in skin and results in the increase of the permeation coefficient $(17,18)$. In addition, IPM is commonly selected for the preparation of MEs due to its well-known permeation enhancing properties and biocompatibility. Surfactant selection is critical for the successful formulation of MEs as it contributes to the reduction of interfacial tension by forming a film at the oil-water interface (19). In the present study, PG displayed a higher solubility in Cremophor-EL (40.193 mg/ml; Table II) compared with Tween-80 (28.051 mg/ml; Table II). Therefore, CremophorEL was selected for use in further studies due to its solubility profile and its low toxicity level as a non-ionic surfactant. Furthermore, its aqueous solution demonstrated good solubility of PG. The highest solubility of PG among the cosurfactants was observed in propylene glycol $(48.714 \mathrm{mg} / \mathrm{ml}$; Table II). In addition, propylene glycol has the ability to form MEs with IPM and CremophorEL. Taken together, IPM, CremophorEL and propylene glycol were used as the oil phase, surfactant and cosurfactant, respectively, for the formulation of PG-loaded MEs.

Construction of pseudo-ternary phase diagrams. In the present study, phase diagrams were constructed to select the concentration range of components for the microemulsions, and the marked areas indicate the clear O/WMEregion (Fig. 1). A close relationship between the hydration effect of the stratum corneum and the dermal permeation has previously been reported (20), and the thermodynamic activity of drugs in MEs was a significant driving force behind the release and penetration of drugs into the skin $(20,21)$. The thermodynamic activity of drugs in ME with lower surfactant mixture content was demonstrated to be an important driving force for the release and the penetration of the drug into skin $(22,23)$. The 
Table I. Compositions and drug loadings of ME formulations.

\begin{tabular}{lccccc}
\hline Formulation & IPM $(\%)$ & CremophorEL $(\%)$ & Propyleneglycol (\%) & Water (\%) & Drug loading $(\%)$ \\
\hline ME1 & 4 & 33 & 11 & 52 & 1 \\
ME2 & 3 & 27 & 9 & 61 & 1 \\
ME3 & 5 & 30 & 10 & 55 & 1 \\
ME4 & 5 & 24 & 8 & 63 & 1 \\
ME5 & 7 & 21 & 7 & 65 & 1 \\
\hline
\end{tabular}

ME, microemulsion; IPM, isopropyl myristate.

Table II. Saturation solubility of phenylethanoid glycoside in different oils, surfactants and co-surfactants.

\begin{tabular}{lc}
\hline Solvent & Solubility $(\mathrm{mg} / \mathrm{ml})$ \\
\hline Ethyl alcohol & $32.285 \pm 0.39$ \\
Propylene glycol & $48.714 \pm 0.51$ \\
Glycerin & $40.561 \pm 0.73$ \\
PEG 400 & $43.193 \pm 0.28$ \\
Cremophor EL & $40.193 \pm 0.34$ \\
Tween 80 & $28.051 \pm 0.14$ \\
Isopropyl myristrate & $0.0125 \pm 0.021$ \\
Oleic acid & $0.0034 \pm 0.037$ \\
Liquid paraffin & $0.0012 \pm 0.061$ \\
\hline
\end{tabular}

decreased concentration of surfactant in dispersed systems may also increase the rate of drug release and its permeation in the skin $(24,25)$. Thus, the water content ranged between 50 and $70 \%$, while that of the surfactant and cosurfactant ranged between 20 and $45 \%$. The microemulsion domain was determined by visual inspection for clarity and fluidity. The rest of the region in the phase diagrams represents turbid and conventional emulsions based on visual observation (Fig. 1). No liquid crystalline structure was observed using the cross polarizer.

The area of the $\mathrm{O} / \mathrm{W} \mathrm{ME}$ region was expanded following the increase of the $\mathrm{Km}$ value (Fig. 1). These findings were consistent with previous studies, which demonstrated that a higher level of surfactant reduces interfacial tension of the colloidal solution more effectively, thereby improving the fluidity of the interface and increasing the entropy of the system $(21,24)$. Thus, it is reasonable to speculate that the effect of CremophorEL on ME regions depends on the other ME components, especially the cosurfactant. The combination of short to medium carbon chain length alcohols, including propylene glycol, with single chain surfactants may lower interfacial tension due to increased fluidity at the interface. Additionally, medium carbon chain length alcohols may induce elevation of miscibility of the aqueous and oily phases due to the partitioning behavior between the two phases. Finally, in the presence of a $\mathrm{Km}$ ratio of $3: 1$, the ratio of the surfactant mixture to oil was 1:9 or $2: 8$. MEs prepared with a ratio of surfactant mixture to oil $>2: 8$ were turbid and instable (Fig. 2). The final compositions of ME1-5 are listed in Table I.
$M E$ preparation and characterization. The physical properties of ME1-5 are listed in Table III. The disperse systems were macroscopically identical, homogeneous, and transparent, without any precipitates. Mean droplet sizes of these MEs ranged between 30.56 and $79.21 \mathrm{~nm}$ (Fig. 3; Table III), with polydispersity-index values ranging between 0.187 and 0.321 (Table III). This indicated a uniform ME with a narrow size distribution. In addition, droplet size increased in proportion with the oil content, but decreased with surfactant mixture content. This phenomenon may be attributed to the expansion of the ME oil core, and the high surfactant concentration forcefully reducing the oil-water interfacial tension to reduce droplet size. Furthermore, ME viscosity was elevated as a result of increased surfactant level and decreased water. The $\mathrm{pH}$ values of all MEs fell within the appropriate range of 5.0-7.5 (Table III) and so were compatible with that of the skin tissues. Regarding physical stability, no phase separation, breaking or drug precipitation were observed in any of the tested MEs, indicating that the MEs possessed satisfactory thermodynamic stability against centrifugation. The dye solubility test revealed that a water-soluble dye (methyl orange) distributed uniformly throughout the ME system, which implied that the formed ME was of $\mathrm{O} / \mathrm{W}$ type (data not shown). The micrographs revealed separate single ME5 droplets with a spherical outline (Fig. 4).

Exvivo skin permeation study. The permeation profiles of all the tested vehicles followed zero-order release kinetics $(26,27)$ and ME5 provided the highest transdermal flux of $P G$ (86.795 $\pm 0.23 \mu \mathrm{g} / \mathrm{cm}^{-2} / \mathrm{h}^{-1}$; Fig. 5; Table III). In the present study, the effects of oil content and surfactant mixture on the skin permeation of PG were determined. The results indicated that surfactants enhance skin permeation by increasing membrane fluidity, drug solubilization, and the extraction of lipid from the stratum corneum. The permeation rate of ME5 $\left(86.795 \pm 0.23 \mu \mathrm{g} / \mathrm{cm}^{-2} / \mathrm{h}^{-1}\right)$ was significantly increased compared with the permeation rates of ME1 $(69.57 \pm 0.78, \mathrm{P}=0.0094)$, ME2 (44.326 $\pm 0.84, \mathrm{P}=0.0052), \mathrm{ME} 3(79.859 \pm 0.12, \mathrm{P}=0.039)$ and ME4 (58.332 $\pm 0.39 \mu \mathrm{g} / \mathrm{cm}^{-2} / \mathrm{h}^{-1}, \mathrm{P}=0.0063$, Fig. 5; Table III) This was consistent with the results of a previous study, in which the thermodynamic activity of the ME drug cargo may decrease with the increase of surfactant levels $(12,28)$. The viscosity of ME5 (30.43 $\pm 0.12 \mathrm{mPa} . \mathrm{s})$ was lower compared with the other ME1 $(80.51 \pm 0.87 \mathrm{mPa} . \mathrm{s}, \mathrm{P}=0.0076)$, ME2 (47.52 $\pm 0.54 \mathrm{mPa} . \mathrm{s}, \mathrm{P}=0.0089)$, ME3 $(60.49 \pm 1.34 \mathrm{mPa} . \mathrm{s}$, $\mathrm{P}=0.0094)$ and ME4 (43.27 $\pm 0.21 \mathrm{mPa} . \mathrm{s}, \mathrm{P}=0.0059)$. The higher fluidity of IPM might have provided improved mobility 
Table III. Characteristics of ME formulation 1-5 and the skin permeation parameters of phenylethanoid glycoside when delivered by these formulations.

Characteristics of MEs

\begin{tabular}{|c|c|c|c|c|c|}
\hline Formulation & Size $(\mathrm{nm})$ & PDI & $\mathrm{pH}$ & Viscosity (mPa.s) & Jss $\left(\mu \mathrm{g} / \mathrm{cm}^{-2} / \mathrm{h}^{-1}\right)$ \\
\hline ME1 & $79.21 \pm 0.01$ & $0.236 \pm 0.05$ & $7.21 \pm 0.01$ & $80.51 \pm 0.87^{\mathrm{b}}(\mathrm{P}=0.0076)$ & $69.57 \pm 0.78^{\mathrm{b}}(\mathrm{P}=0.0094)$ \\
\hline ME2 & $37.91 \pm 0.42$ & $0.187 \pm 0.03$ & $6.98 \pm 0.01$ & $47.52 \pm 0.54^{\mathrm{b}}(\mathrm{P}=0.0089)$ & $44.326 \pm 0.84^{\mathrm{b}}(\mathrm{P}=0.0052)$ \\
\hline ME3 & $67.71 \pm 0.13$ & $0.243 \pm 0.01$ & $6.83 \pm 0.06$ & $60.49 \pm 1.34^{\mathrm{b}}(\mathrm{P}=0.0094)$ & $79.859 \pm 0.12^{\mathrm{a}}(\mathrm{P}=0.039)$ \\
\hline ME4 & $45.32 \pm 0.04$ & $0.321 \pm 0.01$ & $7.09 \pm 0.05$ & $43.27 \pm 0.21^{\mathrm{b}}(\mathrm{P}=0.0059)$ & $58.332 \pm 0.39^{\mathrm{b}}(\mathrm{P}=0.0063)$ \\
\hline ME5 & $30.56 \pm 0.08$ & $0.197 \pm 0.02$ & $6.54 \pm 0.03$ & $30.43 \pm 0.12(\mathrm{P}<0.0001)$ & $86.795 \pm 0.23(\mathrm{P}<0.00010)$ \\
\hline
\end{tabular}

ME, microemulsion; PDI, polydisparity index; Jss, penetration rate. ${ }^{a} \mathrm{P}<0.05,{ }^{\mathrm{b}} \mathrm{P}<0.01$ vs. permeation rate of ME5.

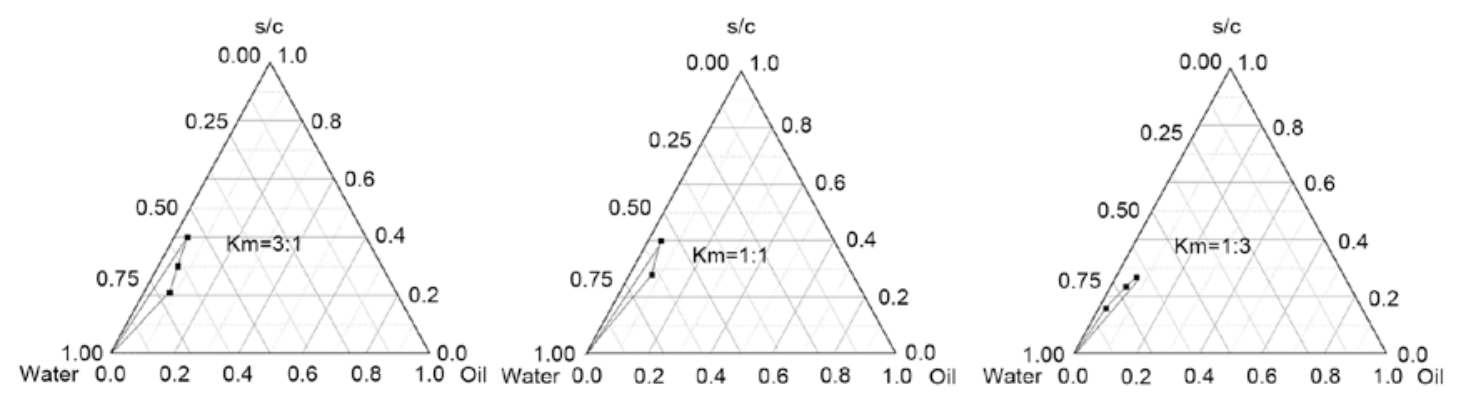

Figure 1. Pseudoternary phase diagrams of microemulsions formulated using different ratios of surfactant (Cremophor EL) to cosurfactant (propylene glycol). $\mathrm{Km}$, weight ratio of surfactant to cosurfactant; s/c, total weight of surfactant and cosurfactant.

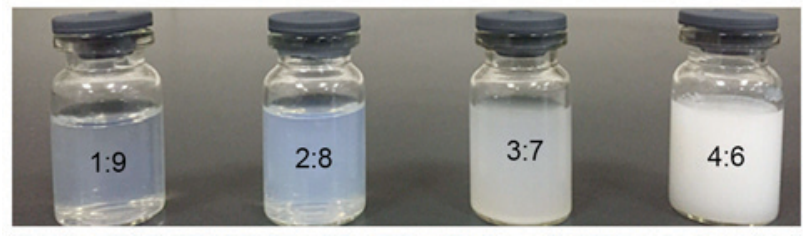

Figure 2. The phase behavior of microemulsions with different ratios of surfactant mixture to oil.

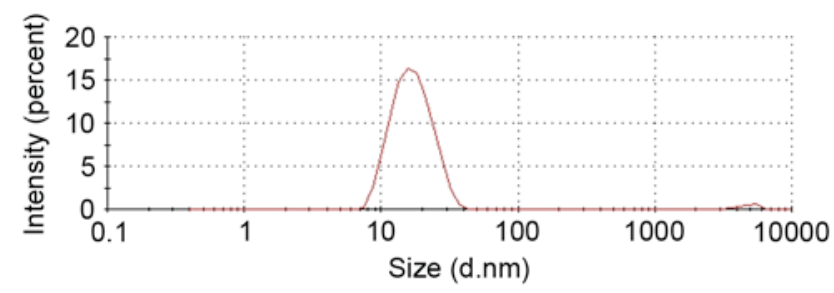

Figure 3. The mean size of phenylethanoid glycosidemicroemulsiondroplets.

for the drug molecules, and resulted in a faster release with a diffusion controlled release mechanism (29). The permeation enhancing effect of IPM may increase the diffusion coefficient of PG-loaded ME in skin tissues, which may result in the increased permeation coefficient (30). Furthermore, the water content of MEs may enhance permeation as hydration of the stratum corneum contributes to the development and widening of channels in the keratin layer and the distortion of the lipid

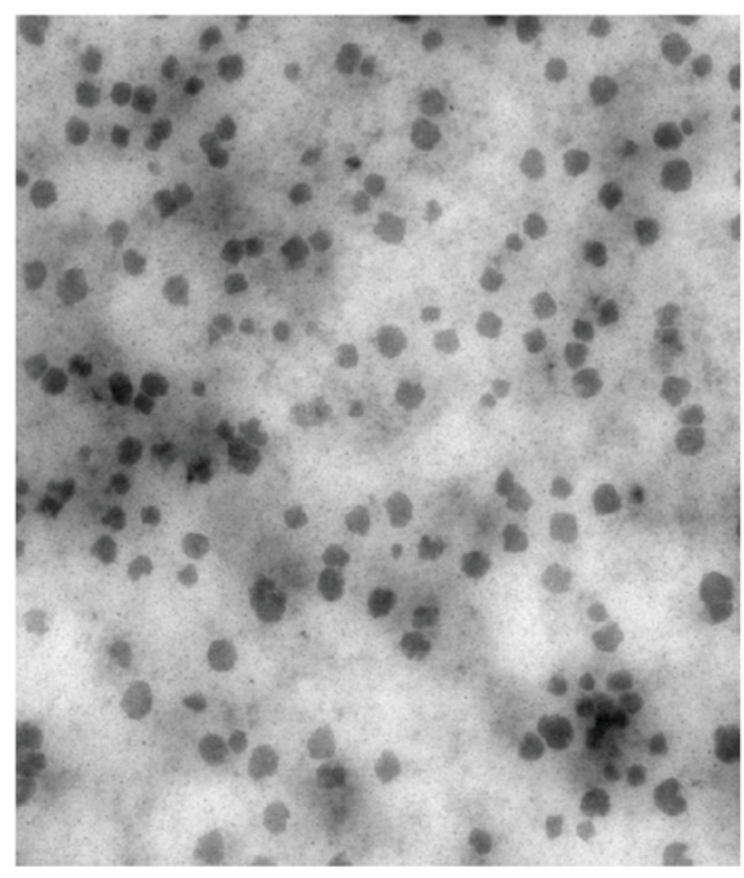

Figure 4. Transmission electron microscopy of microemulsion 5, revealing separate single droplets with spherical outlines. (Magnification x5000).

bilayer (24). In the present study, the water content in ME5 $(65 \%)$ was higher than in the other MEs (Table I), implying that ME5 might induce a higher permeation rate. In addition, the droplet size might affect the permeation profiles, whereby 
Table IV. Accumulative osmotic quantity and the penetration rate of PG when delivered by optimized ME and saline solution.

\begin{tabular}{|c|c|c|c|c|c|c|}
\hline Formulation & Qn-t & $\mathrm{R}^{2}$ & $\begin{array}{c}\text { Jss } \\
\left(\mu \mathrm{g} \cdot \mathrm{cm}^{-2} \cdot \mathrm{h}^{-1}\right)\end{array}$ & $\begin{array}{c}\mathrm{Qn} \\
\left(\mu \mathrm{g} \cdot \mathrm{cm}^{-2}\right)\end{array}$ & $\begin{array}{c}\mathrm{K}_{\mathrm{p}} \\
(\mathrm{cm} / \mathrm{h}) \times 10^{-3}\end{array}$ & $\begin{array}{l}\text { Skin retention } \\
\quad\left(\mu \mathrm{g} \cdot \mathrm{cm}^{-2}\right)\end{array}$ \\
\hline PG saline solution & $\mathrm{Qn}=138.40 \mathrm{t}+190.78$ & 0.9779 & $\begin{array}{c}138.40 \pm 1.56 \\
(\mathrm{P}<0.0001)\end{array}$ & $\begin{array}{c}2288.63 \pm 20.9 \\
(\mathrm{P}<0.0001)\end{array}$ & $\begin{array}{c}5.41 \pm 0.12 \\
(\mathrm{P}<0.0001)\end{array}$ & $\begin{array}{l}93.25 \pm 1.49 \\
(\mathrm{P}<0.0001)\end{array}$ \\
\hline Optimized ME & $Q n=242.49 t+67.18$ & 0.9976 & $\begin{array}{c}242.49 \pm 2.31^{b} \\
(P=0.0071)\end{array}$ & $\begin{array}{c}4149.650 \pm 37.3^{b} \\
(P=0.0039)\end{array}$ & $\begin{array}{l}8.87 \pm 0.25^{\mathrm{a}} \\
(\mathrm{P}=0.041)\end{array}$ & $\begin{array}{c}174.07 \pm 1.51^{\mathrm{a}} \\
(\mathrm{P}=0.029)\end{array}$ \\
\hline
\end{tabular}

${ }^{\mathrm{a}} \mathrm{P}<0.05,{ }^{\mathrm{b}} \mathrm{P}<0.01$ vs. $\mathrm{PG}$ saline solution. Jss, penetration rate; $\mathrm{PG}$, phenylethanoid glycoside; $\mathrm{ME}$, microemulsion; $\mathrm{K}_{\mathrm{p}}$, permeability coefficient; $\mathrm{Q}_{\mathrm{n}}$, the accumulated amount at a given $t$, time.

Table V. Stability analysis results.

\begin{tabular}{|c|c|c|c|c|c|}
\hline \multirow[b]{2}{*}{ Formulation } & \multirow[b]{2}{*}{ Size (nm) } & \multicolumn{2}{|c|}{$\begin{array}{l}\text { Characteristics of } \\
\text { microemulsions }\end{array}$} & \multicolumn{2}{|c|}{ Content } \\
\hline & & PDI & $\mathrm{pH}$ & Viscosity (mPa.s) & $\%$ \\
\hline Before storage & $35.56 \pm 0.06$ & $0.187 \pm 0.03$ & $6.54 \pm 0.02$ & $31.43 \pm 0.10$ & 99.76 \\
\hline After storage & $36.04 \pm 0.04$ & $0.196 \pm 0.01$ & $6.07 \pm 0.02$ & $29.79 \pm 0.08$ & 99.52 \\
\hline
\end{tabular}

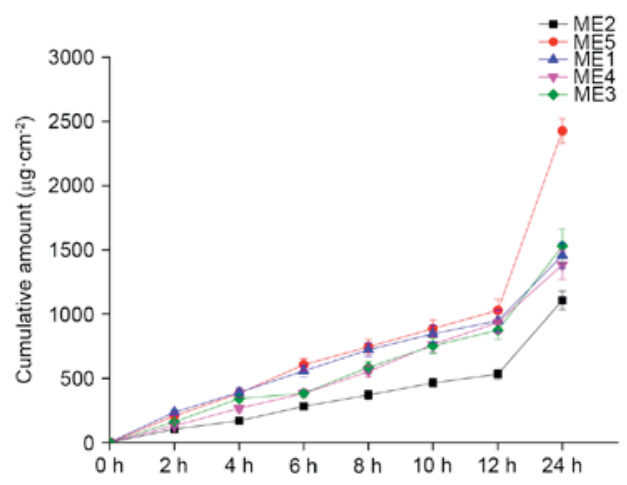

Figure 5. In vitro skin permeation profiles of PG from ME formulations 1-5. PG loading was $1 \%(\mathrm{w} / \mathrm{w})$. PG, phenylethanoid glycoside; ME, microemulsion.

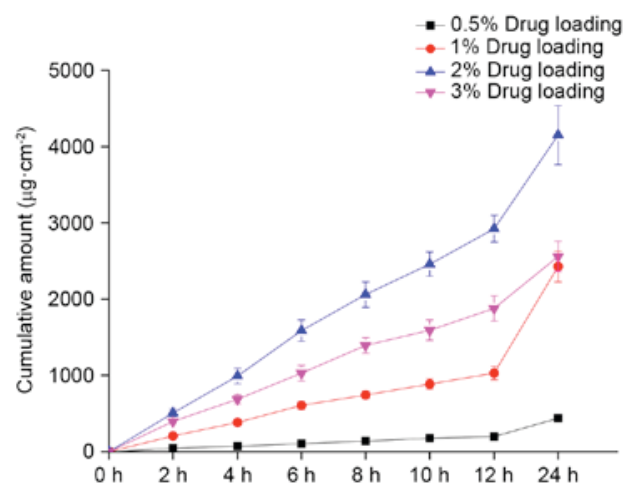

Figure 6. In vitro skin permeation profiles of PG from formulation 5, with PG loading of $0.5,1.0,2.0$, and $3.0 \%(\mathrm{w} / \mathrm{w})$. PG, phenylethanoid glycoside.

ME with smaller droplets tended to increase skin permeation. Taken together, ME5 formulated with the appropriate

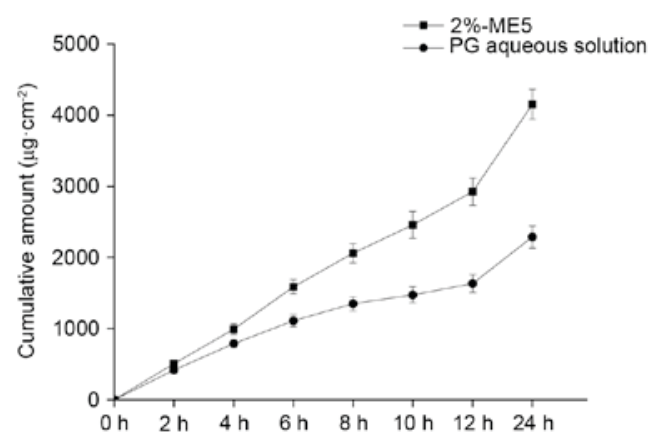

Figure 7. Skin permeation profiles of $P G$ delivered by the optimized ME5 formulation, compared with PG delivered by saline solution. PG, phenylethanoid glycoside; ME5, microemulsion 5.

amount of surfactant mixture (28\%) and water (65\%), with small droplet size $(30.56 \mathrm{~nm})$ and low viscosity (30.43 mPa.s), demonstrated improved permeability compared with the other ME formulations.

The cumulative amount of PG that penetrated the skin increased with drug loading (Fig. 6). In addition, increased drug solubility contributes to the promotion of transdermal drug delivery $(24,31)$. For ME5, a 6.9-fold increase in transdermal flux was noticed in the presence of $2 \%$ drug loading (242.49 $\mu \mathrm{g} / \mathrm{cm}^{-2} / \mathrm{h}^{-1}$; Fig. 6) compared with $0.5 \%$ drug loading (35.504 $\mu \mathrm{g} / \mathrm{cm}^{-2} / \mathrm{h}^{-1}$; Fig. 6). However, due to physical instability at $3.0 \%$ drug loading, resulting in the crystallization of PG during storage, the final loading of PG in ME5 was 2.0\%.

Comparisons between the optimized $\mathrm{ME}$ and the $\mathrm{PG}$ aqueous solution are displayed in Table IV. A 1.68-fold increase in the skin permeation rate of PG was observed in the optimized ME group compared with the PG saline group (Fig. 7). The cumulative amount of PG in the optimized ME group $\left(4149.650 \pm 37.3 \mu \mathrm{g} \cdot \mathrm{cm}^{-2}\right)$ was significantly increased compared 
with the PG saline group $\left(2288.63 \pm 20.9 \mu \mathrm{g} \bullet \mathrm{cm}^{-2}, \mathrm{P}=0.0039\right.$; Table IV) The permeability coefficient indicated that the optimized ME $(8.87 \pm 0.49 \mathrm{~cm} / \mathrm{h}$; Table IV) was also increased compared with the PG saline solution $\left[5.41 \pm 0.12(\mathrm{~cm} / \mathrm{h}) \times 10^{-3}\right.$; $\mathrm{P}=0.041$; Table IV], suggesting that optimized ME is a more effective carrier of $\mathrm{PG}$ than saline. According to previous reports $(16,32)$, high concentration $(2 \%)$ of $\mathrm{PG}$ in in MEs resulted in a high concentration gradient, which contributed to the permeation of PG into the skin. MEs may act as drug reservoirs, with the drug being released from the inner phase to the outer phase and then onto the skin. Furthermore, the MEs may affect the stratum corneum structure and reduce the diffusional barrier by acting as a permeation enhancer $(26,33)$.

Skin retention study. The optimized $\mathrm{ME}$ in the present study demonstrated a higher drug deposition capacity than the PG aqueous solution $\left(174.07 \pm 1.51 \mu \mathrm{g} \cdot \mathrm{cm}^{-2}\right.$ vs. $93.25 \pm 1.49 \mu \mathrm{g} \cdot \mathrm{cm}^{-2}$; $\mathrm{P}=0.029$, Table IV). This maybe associated with the faster release of $\mathrm{PG}$ from the optimized $\mathrm{ME}$, resulting in elevated drug penetration into the skin, or to the higher solubility of the optimized ME compared with the PG saline solution. Due to this effect, an increased amount of PG is retained in the skin. The optimized ME5 formulation demonstrated higher skin retention than the PG saline solution (30). The optimized ME was also superior to the PG saline solution the terms of skin permeation profiles and skin retention (Table IV). Taken together, the optimized ME delivered PG more effectively than the PG saline solution.

Stability study. Stability studies were performed to detect any changes in $\mathrm{pH}$, droplet size, and drug content. Optimized ME was physically stable $(16,29)$ retaining homogeneity and demonstrating no phase separation after 3 months. No major changes were observed in the droplet size and degradation of PG within 3 months. The centrifuge tests demonstrated that optimized ME is physically stable. No significant changes were recorded in the stored ME, but there was a slight decrease in viscosity. This decrease may be due to loss of water during storage. Three months later, no difference was noticed in the $\mathrm{pH}$, droplet size, drug content and viscosity between $\mathrm{ME}$ stored at $2-8^{\circ} \mathrm{C}$ and at room temperature (Table V).

Skin sensitivity test. The intensity criterion of skin irritation followed the protocol, and scores of $<0.5$ meant no irritation, 0.5-3 slight irritation, 3-6 moderate irritation, and $>6$ severe irritation. Little visible irritation was observed in animals treated with optimized ME. The average response scores of skin irritation for saline solution with 2\% (w/w) PG were $0.1 \pm 0.05,0.1 \pm 0.13$, and $0.1 \pm 0.07$ at 24,48 and $72 \mathrm{~h}$, respectively. For the optimized ME, the scores were $0.28 \pm 0.02,0.16 \pm 0.11$ and $0.15 \pm 0.08$ at 24,48 and $72 \mathrm{~h}$, respectively. Despite a slight increase in the scores, optimized ME did not appear to irritate the skin. For these reasons, PG treatment with optimized ME appears to be safe.

A novel ME was prepared using IPM as the oil phase, CremophorEL as the surfactant and propyleneglycolas the cosurfactant. This formulation was characterized by a transparent appearance, low viscosity, a spherically uniform distribution, stable physical and chemical properties, as well as good stability. Compared with saline solution, a 1.68-fold increase was noted in the skin permeation rate of PG for optimized microemulsion. The cumulative amount of PG in the microemulsion $\left(4149.650 \pm 37.3 \mu \mathrm{g} \cdot \mathrm{cm}^{-2}\right)$ was significantly higher than that of $\mathrm{PG}$ in the saline solution $\left(2288.63 \pm 20.9 \mu \mathrm{g} \cdot \mathrm{cm}^{-2}\right)$. Furthermore, the permeability coefficient indicated optimized microemulsion was a more efficient carrier for transdermal delivery of $\mathrm{PG}$ than the control solution $\left(8.87 \pm 0.49 \mathrm{~cm} / \mathrm{h} \times 10^{-3}\right.$ vs. $\left.5.41 \pm 0.12 \mathrm{~cm} / \mathrm{h} \times 10^{-3}\right)$, the skin permeation ability of $\mathrm{PG}$ was significantly increased by this $\mathrm{ME}$, which may be due to the special characteristics of MEs.

\section{Acknowledgements}

The present study was supported by the National Nature Science Foundation of China (grant no. 81560629), the National Nature Science Foundation of China (grant no. 81360670) and the Xinjiang Scientific Innovation Program (grant no. 201472105).

\section{References}

1. Qiu Y, Chen M, Su M, Xie G, Li X, Zhou M, Zhao A, Jiang J and Jia W: Metabolic profiling reveals therapeutic effects of Herba Cistanches in an animal model of hydrocortisone-induced'kidney-deficiency syndrome'. Chin Med 3: 3, 2008.

2. Fu G, Pang $\mathrm{H}$ and Wong YH: Naturally occurring phenylethanoid glycosides: Potential leads for new therapeutics. Curr Med Chem 15: 2592-2613, 2008.

3. Saracoglu I, Harput US, Inoue M and Ogihara Y: New phenylethanoid glycosides from Veronica pectinata var. glandulosa and their free radical scavenging activities. Chem Pharm Bull (Tokyo) 50: 665-668, 2002.

4. Yamaguchi K, Mitsui T, Aso $\mathrm{Y}$ and Sugibayashi K: Structure-permeability relationship analysis of the permeation barrier properties of the stratum corneum and viable epidermis/dermis of rat skin. J Pharm Sci 97: 4391-4403, 2008.

5. Tandel H, Raval K, Nayani A and Upadhay M: Preparation and evaluation of cilnidipine microemulsion. J Pharm Bioallied Sci 4 (Suppl 1): S114-S115, 2012.

6. M El Maghraby G: Microemulsions as transdermal drug delivery systems. Curr Nanoscience 8: 504-511, 2012.

7. Mou D, Chen H, Du D, Mao C, Wan J, Xu H and Yang X: Hydrogel-thickened nanoemulsion system for topical delivery of lipophilic drugs. Int J Pharm 353: 270-276, 2008.

8. Barot BS, Parejiya PB, Patel HK, Gohel MC and Shelat PK: Microemulsion-based gel of terbinafine for the treatment of onychomycosis: Optimization of formulation using D-optimal design. AAPS PharmSciTech 13: 184-192, 2012.

9. Tsai YH, Lee KF, Huang YB, Huang CT and Wu PC: In vitro permeation and in vivo whitening effect of topical hesperetin microemulsion delivery system. Int J Pharm 388: 257-262, 2010.

10. Li G, Fan Y, Li X, Wang X, Li Y, Liu Y and Li M: In vitro and in vivo evaluation of a simple microemulsion formulation for propofol. Int J Pharm 425: 53-61, 2012.

11. Yang JH, Wu SS, Xu HH, Yan Y, Ju B, Zhu D, Liang X and Hu J: Inhibitory effects of phenylethanoid glycosides on melanin synthesis in cultured human epidermal melanocytes. Int J Clin Exp Med 9: 18019-18025, 2016.

12. Shen LN, Zhang YT, Wang Q, Xu L and Feng NP: Preparation and evaluation of microemulsion-based transdermal delivery of total flavone of rhizoma arisaematis. Int J Nanomedicine 9: 3453-3464, 2014.

13. Draize JH, Woodard G and Calvery HO: Methods for the study of irritation and toxicity of substances applied topically to the skin and mucous membranes. J Pharmacol Exp Therapeutics 82: 377-390, 1944

14. Sahoo S, Pani NR and Sahoo SK: Microemulsion based topical hydrogel of sertaconazole: Formulation, characterization and evaluation. Colloids Surf B Biointerfaces 120: 193-199, 2014.

15. Paolino D, Ventura CA, Nisticò S, Puglisi G and Fresta M: Lecithin microemulsions for the topical administration of ketoprofen: Percutaneous adsorption through human skin and in vivo human skin tolerability. Int J Pharm 244: 21-31, 2002. 
16. Butani D, Yewale C and Misra A: Amphotericin B topical microemulsion: Formulation, characterization and evaluation. Colloids Surf B Biointerfaces 116: 351-358, 2014.

17. Acosta EJ, Nguyen T, Witthayapanyanon A, Harwell JH and Sabatini DA: Linker-based bio-compatible microemulsions. Environ Sci Technol 39: 1275-1282, 2005.

18. Chen H, Mou D, Du D, Chang X, Zhu D, Liu J, Xu H and Yang X: Hydrogel-thickened microemulsion for topical administration of drug molecule at an extremely low concentration. Int J Pharm 341: 78-84, 2007.

19. Bali V, Ali M and Ali J: Study of surfactant combinations and development of a novel nanoemulsion for minimising variations in bioavailability of ezetimibe. Colloids Surf B Biointerfaces 76 : 410-420, 2010

20. Changez $M$ and Varshney M: Aerosol-OT microemulsions as transdermal carriers of tetracaine hydrochloride. Drug Dev Ind Pharm 26: 507-512, 2000

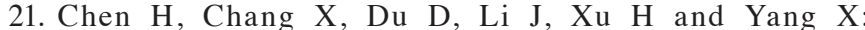
Microemulsion-based hydrogel formulation of ibuprofen for topical delivery. Int J Pharm 315: 52-58, 2006.

22. Shakeel F, Baboota S, Ahuja A, Ali J, Aqil M and Shafiq S: Nanoemulsions as vehicles for transdermal delivery of aceclofenac. AAPS PharmSciTech 8: E104, 2007.

23. Zhang YT, Zhao JH, Zhang SJ, Zhong YZ, Wang Z, Liu Y, Shi F and Feng NP: Enhanced transdermal delivery of evodiamine and rutaecarpine using microemulsion. Int J Nanomedicine 6: 2469-2482, 2011.

24. Sintov AC: Transdermal delivery of curcumin via microemulsion. Int J Pharm 481: 97-103, 2015.

25. Zhao L, Wang Y, Zhai Y, Wang Z, Liu J and Zhai G: Ropivacaine loaded microemulsion and microemulsion-based gel for transdermal delivery: Preparation, optimization, and evaluation. Int J Pharm 477: 47-56, 2014.
26. Moreno MA, Frutos P and Ballesteros MP: Lyophilized lecithin based oil-water microemulsions as a new and low toxic delivery system for amphotericin B. Pharm Res 18: 344-351, 2001.

27. Panapisal V, Charoensri S and Tantituvanont A: Formulation of microemulsion systems for dermal delivery of silymarin. AAPS PharmSciTech 13: 389-399, 2012.

28. Qi J, Zhuang J, Wu W, Lu Y, Song Y, Zhang Z, Jia J and Ping Q: Enhanced effect and mechanism of water-in-oil microemulsion as an oral delivery system of hydroxysafflor yellow A. Int J Nanomedicine 6: 985-991, 2011.

29. Fouad SA, Basalious EB, El-Nabarawi MA and Tayel SA: Microemulsion and poloxamer microemulsion-based gel for sustained transdermal delivery of diclofenac epolamine using in-skin drug depot: In vitro/in vivo evaluation. Int J Pharm 453: 569-578, 2013.

30. Gundogdu E, Alvarez IG and Karasulu E: Improvement of effect of water-in-oil microemulsion as an oral delivery system for fexofenadine: In vitro and in vivo studies. Int J Nanomedicine 6: 1631-1640, 2011

31. Zhao JH, Ji L, Wang H, Chen ZQ, Zhang YT, Liu Y and Feng NP: Microemulsion-based novel transdermal delivery system of tetramethylpyrazine: Preparation and evaluation in vitro and in vivo. Int J Nanomedicine 6: 1611-1619, 2011

32. Zhu W, Yu A, Wang W, Dong R, Wu J and Zhai G: Formulation design of microemulsion for dermal delivery of penciclovir. Int $J$ Pharm 360: 184-190, 2008.

33. Wang Z, Mu HJ, Zhang XM, Ma PK, Lian SN, Zhang FP, Chu SY, Zhang WW, Wang AP, Wang WY and Sun KX: Lower irritation microemulsion-based rotigotine gel: Formulation optimization and in vitro and in vivo studies. Int J Nanomedicine 10: 633-644, 2015. 